\title{
Prospective Comparative Study between Retroperitoneoscopic and Hand-Assisted Laparoscopic Approach for Radical Nephrectomy
}

\author{
Marcos Tobias-Machado, Pedro I. Ravizzini, Leonardo O. Pertusier, Eduardo Pedroso, Eric R. \\ Wroclawski
}

Division of Urologic Oncology and Laparoscopy (MTM, PIR, LOP, EP, ERW), ABC Medical School, Santo Andre, Sao Paulo, Research and Educational Institute of Albert Einstein Jewish Hospital (MTM, ERW) Sao Paulo, and Brazilian Institute for Cancer Control (MTM, ERW), Sao Paulo, SP, Brazil

\begin{abstract}
Objective: No consensus has yet been established regarding the best minimally invasive access for radical ablation of renal tumors. Our objective was to prospectively compare the surgical results and oncologic management of two currently used endoscopic techniques.

Materials and Methods: Over a four-year period, 50 patients with renal tumors and clinical stage T1b-T2, smaller than 12 $\mathrm{cm}$, underwent a radical nephrectomy at two reference institutions, 25 underwent retroperitoneoscopic radical nephrectomy (RRN) and 25 a hand-assisted laparoscopic radical nephrectomy (HALRN). Mean follow-up of both cohorts was 50 months. Operative parameters and oncological management were compared.

Results: The mean operative time was $180 \mathrm{~min}$ in RRN and $108 \mathrm{~min}$ in $\operatorname{HALRN}(\mathrm{p}<0.001)$. The time required to access the renal pedicle in RRN was 30 min. and in HALRN 40 min., Learning curve was shorter in HALRN than RRN. Mean blood loss was $100 \mathrm{~mL}$ in RRN and $242 \mathrm{~mL}$ in HALRN. Mean incision size for specimen retrieval in RRN was $6.5 \mathrm{~cm}$ and in HALRN $7.5 \mathrm{~cm}$. One patient with intra operative occurrence of ascites and subsequent pathological stage pT2N0M0 grade 3 operated via HALRN, had neoplasic implants in the Hand-port incision 3 months after surgery followed by death 4 months after recurrence. One patient, with pathological stage pT3N0M0 grade 3 in RRN had metastasis after 36 months. Conclusion: Both, RRN and HALRN techniques are accepted minimally invasive options for endoscopic radical nephrectomy with equivalent long term oncological outcome in the treatment of renal tumors.
\end{abstract}

Key words: kidney neoplasms; surgery; nephrectomy; laparoscopy

Int Braz J Urol. 2009; 35: 284-92

\section{INTRODUCTION}

Laparoscopic radical nephrectomy has been accepted as a treatment of choice for renal tumors with stage T1-T2 smaller than $8 \mathrm{~cm}$ when radical surgery is indicated (1-4).

There are three different minimally invasive approaches described for endoscopic ablation of the kidney: laparoscopic, retroperitoneoscopic and hand - assisted laparoscopic techniques. Each technique offers advantages and disadvantages, which can be adopted depending on the individual preferences of each surgeon. There are only a few reported prospective studies using different techniques that rigorously compare the benefits of one approach over the other. 
The retroperitoneoscopic technique has won increasing world acceptance. Through this access, there is no intra-peritoneal manipulation, therefore reducing the chance of iatrogenic abdominal lesions (3).

The hand-assisted laparoscopic radical nephrectomy (HALRN), initially described in 1994 had not gained popularity until the introduction of hand-port devices for maintenance of the pneumoperitoneum. The potential advantages of this technique are the adjunct of tactile sensation of the laparoscopic procedure, increased orientation, manual dissection access and retraction of anatomic structures. It is particularly useful when an incision is necessary to remove an intact surgical specimen or when a large size tumor has to be resected $(5,6)$.

The aim of this study was to prospectively compare operative and oncologic outcomes of both techniques as different options for radical surgical treatment of renal cell carcinomas (RCC).

\section{MATERIAL AND METHODS}

In a period from February 1999 to February 2003, 25 retroperitoneoscopic radical nephrectomies (RRN) and 25 HALRN were performed in the two institutions. Both groups were comparable regarding demographic data (Table-1). The patients presented good performance status (ASA score I or II) and no formal contraindications to the laparoscopic approach. Fifty patients with the diagnosis of a solid renal mass, larger than $4 \mathrm{~cm}$ and smaller or equal to $12 \mathrm{~cm}$, with clinical stage T1b-T2N0M0 RCC determined by abdominal CT scan and chest X-ray, were prospectively included. Surgical procedures including cases of learning curve were performed at 2 regional referral teaching hospitals, by a single surgeon in each institution with experience in laparoscopic procedures. The two techniques were performed in accordance with the techniques previously described in literature $(1,3)$. In the RRN group, there were 10 male and 15 female patients, with a mean age of 60 years (range 45 to 75 ), and a mean body mass index (BMI) of 24 (range 17 to 32 ). The mean size of the tumors in the pre-operative tomographic study was $7 \mathrm{~cm}$ (range 4 to 11) (Table-1). Nineteen (76\%) of the patients had a clinical stage T1N0M0, and 6 (24\%), T2N0M0. In the HALRN group, there were 11 male and 14 female patients, with a mean age of 55.5 years (range 38 to 77) and mean BMI of 23 (range 18 to 30). Mean tumor size in tomographic study was of $7 \mathrm{~cm}$ (range 4 to 12) (Table-1). Sixteen (64\%) of the patients presented clinical stage T1N0M0, and 9 (36\%) T2NOM0. The two groups were compared according to operative time, learning curve (analyzing the first 15 cases, and remaining 10 separately), time of access to renal pedicle, estimated blood loss, need for painkillers postoperatively, size of incision for specimen retrieval, complications, need for blood transfusion, specimen histopathologic analysis and oncologic management during a mean follow-up time of 30 months.

Statistical analysis used was Student-t-test and Chi-square test. The difference was considered significant at $\mathrm{p}<0.05$.

\section{RESULTS}

\section{Intra-operative Data}

Mean operative time was $180 \mathrm{~min}$. (range 120 to 240 ) in the RRN group, and 108 min (range 80 to $140)$ in the HALRN $(\mathrm{p}<0.001)$.

In the RRN group, the mean operative time for the first 15 procedures was $220 \mathrm{~min}$. (range 100 to 140 ), and for the remaining $15,96 \mathrm{~min}$. (range 80 to 120). The time of access to the renal pedicle after installing the portals was on average $30 \mathrm{~min}$. (20 to 60) for the RRN, and $40 \mathrm{~min}$. (30 to 75) for the HALRN group.

Adrenalectomy was executed in 18 patients (72\%) of the 8 RRN group, and $12(48 \%)$ of the HALRN group. Mean estimated blood loss was of $100 \mathrm{~mL}$ (range 30 to 200) in the RRN group, and 242 $\mathrm{mL}$ (range 160 to 320 ) in the HALRN group. Blood intra-operative transfusions were not required in any of the procedures in either group. Mean incision size for specimen retrieval of the RRN group was $6.5 \mathrm{~cm}$ ( 5 to 9), and for the HALRN group $7.5 \mathrm{~cm}$ (Table-2).

\section{Intra-operative Complications}

Complications occurred in 5 patients in the RRN group (20\%). One patient (4\%) developed a 
Table 1 - Demographic data comparing retroperitoneoscopic radical nephrectomy (RRN) and hand-assisted laparoscopic radical nephrectomy (HALRN).

\begin{tabular}{lccc}
\hline & RRN & HALRN & p Value \\
\hline Number of patients & 25 & 25 & $\mathrm{p}=0.12$ \\
Age in years (mean) & $45-75(60) \pm 8.5$ & $38-77(55.5) \pm 11.5$ & $\mathrm{p}=0.39$ \\
Body mass index (index) & $17-32(23) \pm 3.8$ & $18-30(24) \pm 4.4$ & $\mathrm{p}=1.0$ \\
Tumor side (right/left) & $16 / 9$ & $13 / 12$ & $\mathrm{p}=0.19$ \\
Tumor location (upper/median/lower) & $7 / 7 / 11$ & $8 / 7 / 10$ & $\mathrm{p}=0.16$ \\
Tumor size in cm (mean) & $4-11(7) \pm 3.0$ & $4-12(7) \pm 3.5$ & $\mathrm{p}=1.0$ \\
Clinical Stage & 19 & 16 & \\
$\quad$ T1N0M0 & 6 & 9 & $\mathrm{p}=0.54$ \\
$\quad$ T2N0M0 & & & $\mathrm{p}=0.54$ \\
\hline \multicolumn{1}{c}{} \\
\hline
\end{tabular}

hernia at the site of the incision for specimen retrieval. Four patients $(16 \%)$ had a major complication: one pneumothorax (treated by thoracic tube drainage), three vascular intra-operative lesions that were all controlled endoscopically.

In the HALRN group we observed complications in $4(16 \%)$ patients. Three $(12 \%)$ presented minor complications: two (8\%) anterior abdominal wall hematomas (one patient, needed a second post-operative day blood transfusion) and one (4\%) incisional hernia at one of the $10 \mathrm{~mm}$ port sites. One (4\%) of the patients presented an intra-operative splenic laceration and underwent a hand-assisted laparoscopic splenectomy. There were no conversions to open surgery in any of the groups (Table-3).

\section{Postoperative Data}

Mean time for first deambulation was 6 hours in RRN and 8 hours in HALRN group. Mean postoperative diet reintroduction time was 1.5 and 1.7 days in the RRN and HALRN group respectively. Mean hospital stay was 2.5 (1 to 4 ) days in the RRN group, and 2.2 (2 to 3 ) days in the HALRN group. The mean morphine equivalent intake requirement in the RRN group was $45 \mathrm{mg}$ (range 17 to 120 ) and $55 \mathrm{mg}$ (range 20 to 120) in the HALRN group. None of the patients needed I.V. analgesics after the second day hospital stay. Fourteen patients $(56 \%)$ in the RRN group and $20(75 \%)$ in the HALRN group continued taking paracetamol tablets for more than 2

Table 2 - Intra-operative data comparing retroperitoneoscopic radical nephrectomy (RRN) and hand-assisted laparoscopic radical nephrectomy (HALRN).

\begin{tabular}{lccc}
\hline & RRN & HALRN & p Value \\
\hline Operative time in min (mean) & $120-240(180) \pm 42$ & $80-140(108) \pm 42$ & $\mathrm{p}<0.001$ \\
First 15 patients (mean) & $200-240(220)$ & $100-140(120)$ & \\
Last 10 patients (mean) & $120-160(140)$ & $80-120(96)$ & \\
Time for renal pedicle access in min (mean) & $20-60(30)$ & $30-75(40)$ & \\
Average blood loss in mL (mean) & $30-200(100) \pm 382$ & $160-320(242) \pm 204$ & $\mathrm{p}=0.10$ \\
Need for intra-operative transfusion & 0 & 0 & \\
Number of adrenalectomies & 18 & 12 & \\
Incision size for specimen retrieval in cm (mean) & $5-9(6.5)$ & 7.5 & \\
\hline
\end{tabular}


Table 3 - Surgical complications comparing retroperitoneoscopic radical nephrectomy (RRN) and hand-assisted laparoscopic radical nephrectomy (HALRN).

\begin{tabular}{|c|c|c|c|}
\hline Complications & RRN (\%) & HALRN (\%) & p Value \\
\hline Minor & & & $\mathrm{p}=1$ \\
\hline Incisional hernia & $1(4)$ & $1(4)$ & \\
\hline Subcutaneous hematoma & - & $2(8)$ & \\
\hline Total & $1(4)$ & $3(12)$ & \\
\hline \multicolumn{4}{|l|}{ Major } \\
\hline Pneumothorax & $1(4)$ & - & \\
\hline Vascular lesions & $3(12)$ & - & \\
\hline Spleen laceration & - & $1(4)$ & \\
\hline Conversion & - & - & \\
\hline Total & $1(4)$ & $1(4)$ & \\
\hline Total of complications (mean) & $6(20)$ & $4(16)$ & \\
\hline
\end{tabular}

days. Mean time for convalescence was 3 weeks in the Retroperitoneoscopic group and 4 weeks in the Hand-assisted group (Table-4).

\section{Histopathologic Data}

In all patients a complete and intact removal of the surgical specimen was achieved. The diagnosis of renal cell carcinoma was confirmed in all patients in the RRN group. In the HALRN group, 23 specimens corresponded to renal cell carcinomas, one presented as an oncocytoma, and the other as a renal adenoma. Pathologic stages in the Retroperitoneoscopic group was a T1N0M0 in 18
(72\%), T2N0M0 in $4(16 \%)$, T3N0M0 in $2(8 \%)$ and T3N1M0 in $1(4 \%)$ of the patients. In the Hand-assisted group, pathologic stages were T1N0M0 in 15 $(60 \%)$, T2N0M0 in $6(24 \%)$ and T3N1M0 in $2(8 \%)$. The mean surgical specimen weight was $310 \mathrm{~g}(95$ to $410 \mathrm{~g}$ ) in the Retroperitoneoscopic group and $482 \mathrm{~g}$ (130 to $800 \mathrm{~g}$ ) in the Hand-assisted group (Table-5).

Negative surgical margins were obtained in all surgical specimens. The RRN group specimens had a larger mean diameter size, $12.2 \mathrm{~cm}$ (8 to 20), and mean tumor size, $6.9 \mathrm{~cm}(3.5$ to $11 \mathrm{~cm})$ compared to the specimens of the HALRN group, which had an average size of $10.3 \mathrm{~cm}$ ( 9 to $22 \mathrm{~cm}$ ) and mean tumors size of $6.4 \mathrm{~cm}$ (4 to $12 \mathrm{~cm})$.

Table 4-Postoperative data comparing retroperitoneoscopic radical nephrectomy (RRN) and hand-assisted laparoscopic radical nephrectomy (HALRN).

\begin{tabular}{lccc}
\hline & RRN & HALRN & p Value \\
\hline Mean time for first deambulation & $6 \mathrm{~h} \pm 0.6$ & $8 \mathrm{~h} \pm 2.3$ & $\mathrm{p}<0.001$ \\
$\begin{array}{l}\text { Mean time for reintroduction of } \\
\text { postoperative diet }\end{array}$ & 1.5 days \pm 0.35 & 1.7 days \pm 0.3 & $\mathrm{p}<0.05$ \\
Hospital stay (mean) & & & \\
Analgesic intake (mean) & $1-4$ days (2.5) \pm 1.1 & $2-3$ days $(2.2) \pm 2.3$ & $\mathrm{p}=0.56$ \\
Mean convalescence time & $17-120 \mathrm{mg}(45) \pm 19.9$ & $20-120 \mathrm{mg}(55) \pm 4.9$ & $\mathrm{p}=0.018$ \\
\hline
\end{tabular}


Table 5 - Hystopathologic data comparing retroperitoneoscopic radical nephrectomy (RRN) and hand-assisted laparoscopic radical nephrectomy (HALRN).

\begin{tabular}{lccc}
\hline & RRN & HALRN & p Value \\
\hline Renal cell carcinoma & 25 & 23 & \\
Specimen weight (mean) & $95-410 \mathrm{~g}(310)$ & $130-800(482)$ & \\
Specimen size (mean) & $8-20 \mathrm{~cm}(12.2)$ & $9-22(10.3)$ & \\
Tumor size (mean) & $3.5-11 \mathrm{~cm}(6.9) \pm 1.3$ & $4-12(6.4) \pm 2.9$ & $\mathrm{p}=0.44$ \\
Pathologic stage & & & $\mathrm{p}=0.50$ \\
T1N0M0 & 18 & 14 & \\
T2N0M0 & 4 & 7 & \\
T3N0M0 & 2 & - & \\
T3N1M0 & 1 & 1 & \\
T3bN1M0 & - & 1 & \\
\hline
\end{tabular}

\section{Oncologic Follow-up}

Mean follow-up time for the RRN and HALRN groups were respectively 48 months (36 to $58 \mathrm{mo}$ ) and 52 months (30 to $66 \mathrm{mo}$ ) respectively. In the RRN group, one patient presenting with pT3N1M0 grade 3 stage developed local recurrence, adrenal and retroperitoneal metastasis, 36 months after surgical resection. At the moment the patient is undergoing interferon therapy, and is still alive 40 months after surgery. There were no deaths in this group.

In the HALRN group, there was one death not-related to the neoplasia. Also, one patient with pT3N1M0 grade 3 stage, presented 4 months after surgery with subcutaneous tumors at one of the 10 $\mathrm{mm}$ laparoscopic ports and Hand-port incision sites. The patient also was found to have another implant site on the anterior abdominal wall, with no relation to surgical incisions. The patient died 4 months after surgery. This patient was diagnosed intra-operatively with ascites and signs of the peritoneal carcinomatosis at the time of HALRN. There were no other cases of disease recurrence in this group.

\section{COMMENTS}

Radical nephrectomy is considered the established treatment for renal cell carcinoma. Several published data suggests that laparoscopic radical nephrectomy for stage T1-2 tumors, smaller than 8 $\mathrm{cm}$, present relative advantages, reducing morbidity compared to the open technique, with better post-operative recovery and shorter return to normal activities $(4,7)$.

The ideal minimally invasive method is not yet widely defined. Radical laparoscopic pure nephrectomy can be performed via a trans-peritoneal and a retro-peritoneal approach. Retroperitoneoscopic radical nephrectomy is currently gaining increasing world acceptance for the treatment of renal tumors $(3,8,9)$.

Hand-assisted laparoscopic radical nephrectomy has been presented as another technical option and, according to some authors, is considered best indicated in selected cases $(10,11)$. Some reports suggest that the choice between endoscopic techniques for renal ablation depends on the experience and preference of the surgeon, as well as the advantages, and disadvantages offered by each technique.

Regarding the indications, the retroperitoneoscopic access has been recommended for patients with previous abdominal surgery. Some authors avoid RRN when the tumor has a large volume, due to its smaller working space. On the other hand, Gill et al. (3) performed retroperitoneoscopic renal surgery in tumors with sizes of up to $12 \mathrm{~cm}$. In our series, we also performed this technique, independently from 
the size of the tumor, as long as the renal pedicle was apparently free of tumor on the CT scan images. For some authors, HALRN constitutes a selective indication for larger tumors. HALRN could also be a more appropriate option for patients with co-morbidities where a shorter operative time would be advised.

Each of the techniques has some limitations and demands some kind of adaptation by surgeon. In the retroperitoneal access, as there is a less significant working space, small CO2 losses may make it difficult to perform surgery $(12,13)$. Moreover, to avoid this problem, the surgeon should develop an adequate expansion of the retroperitoneal space, use rigorously sealed ports, perform optimized laparoscopic aspiration and increase $\mathrm{CO} 2$ replacement speed.

In HALRN the biggest limitation may be a smaller working space for hand insertion in the small abdominal cavities (12).

The main advantages of the retroperitoneoscopic technique are the absence of intra abdominal manipulation, therefore reducing the chance of iatrogenic lesions of the intra-peritoneal organs $(3,4,8,9)$, and the direct access to the renal pedicle allowing early control of the renal artery and vein $(3,9)$. In our series we obtained faster access to the renal pedicle with RRN than with HALRN.

The main advantages for HALRN are a shorter operative time and facilitating the approach to masses of greater volume, especially in large sized kidney tumors. It also facilities, to some extent, vascular control in the case of a major vascular lesion and specimen retrieval at the end of the procedure.

Considering the learning curves for both techniques, HALRN has significantly shorter operative time for the initial cases than any pure laparoscopic technique, but there is less reduction in operating time when the technique is mastered (12). With RRN we observed a definite impact on the learning curve, with significant reduction of operating time, when more experience is acquired. These results explain the larger acceptance of the Hand-assisted technique by surgeons with less experience in laparoscopic surgery. Our first 15 cases, include as learning curve in a sample of 25 patients in each group however, this may be a limitation in our study.

Blood loss in the RRN group was smaller. Reported data are conflicting as we evaluated blood loss on different endoscopic accesses for radical nephrectomy. A possible explanation for our results is that in the HALRN group, blunt dissection was employed throughout the procedure as a means of reducing operating time. In fact, in our data, the HALRN technique showed a significantly shorter operating time, which does not agree with the other author's results. Again, blunt dissection may have had played an important role in reducing operating time but the difference obtained could be partially explained by the largest percentage of adrenalectomies performed in the RRN group.

When the requirement for analgesia and hospital stay was considered, there was no significant difference between both groups. Although it seems logical that the retroperitoneoscopic technique would require a smaller dosage of analgesics than Hand-assisted techniques, some comparative studies failed to demonstrate this advantage $(10,11,13,14)$.

Complications rates in our study were similar for both techniques. The RRN group presented a higher index of vascular lesions than the HALRN group. However, all lesions were controlled laparoscopically and no blood transfusions were necessary in any of the cases. These data are not in agreement with the series reported by other authors where major complications were more frequent for the Hand-assisted method (10,14-16). Minor complications represented by incisional hernia and parietal hematoma, were more frequent in the HALRN group. Parietal hematoma was caused by a partial epigastric arterial lesion at the 10 $\mathrm{mm}$ port site, detected post-operatively. An extensive abdominal wall hematoma was formed and blood transfusion was required in the second post-operative day.

The transverse incision for specimen extraction in the retroperitoneoscopic technique is smaller, and may be applied to the flank, therefore in order to achieve a more aesthetic result. The incision for the Hand-port placement is generally longitudinal or oblique, larger in comparison, and is applied to the anterior abdominal wall, therefore producing a less aesthetic result.

It has only recently been shown that long term oncologic management of patients who have undergone laparoscopic radical nephrectomy is similar to conventional surgery $(17,18)$. In both groups analyzed 
in this study, surgeons opted for an intact specimen removal. Laparoscopic port site tumoral seeding was not observed in any cases from the RRN group over a period of 30 months. In our series, we had a patient, in each group, who presented metastases related to a high histologic grade with lymph node involvement. However, a longer follow-up period would be necessary to evaluate the oncological results related to each technique.

Our results suggest that in general, both techniques are equivalent and feasible in the treatment of renal malignant tumors. The knowledge of both surgical techniques is important for an adequate selection of the optimal minimally invasive access to be used in each case. The hand assisted technique in none of the referral centers was considered useful to introduce the practice of minimally invasive procedures.

\section{CONCLUSION}

Hand-assisted technique showed a relative reduction in operating time, which may be especially useful in more debilitated patients, and a good option for the treatment of large size renal tumors.

Retroperitoneoscopic technique provides early access to vascular control, permitting minimal blood loss. In addition, the removal of the specimen was possible by using a more aesthetic incision.

The comparison of long term oncological data seems to be similar using either one of these two techniques.

\section{CONFLICT OF INTEREST}

None declared.

\section{REFERENCES}

1. Gill IS: Laparoscopic radical nephrectomy for cancer. Urol Clin North Am. 2000; 27: 707-19.

2. Ono Y, Kinukawa T, Hattori R, Yamada S, Nishiyama N, Mizutani K, et al.: Laparoscopic radical nephrectomy for renal cell carcinoma: a five-year experience. Urology. 1999; 53: 280-6.
3. Gill IS, Schweizer D, Hobart MG, Sung GT, Klein EA, Novick AC: Retroperitoneal laparoscopic radical nephrectomy: the Cleveland clinic experience. J Urol. 2000; 163: 1665-70.

4. Allan JD, Tolley DA, Kaouk JH, Novick AC, Gill IS: Laparoscopic radical nephrectomy. Eur Urol. 2001; 40: $17-23$

5. Tierney JP, Oliver SR, Kuminsky RE, Tiley EH, Lucente FC, Boland JP: Laparoscopic radical nephrectomy with intra-abdominal manipulation. Minimal Invas. Ther. 1994; 3: 303-5.

6. Kusminsky RE, Tiley EH, Lucente FC, Boland JP: Laparoscopic staging laparotomy with intra-abdominal manipulation. Surg Laparosc Endosc. 1994; 4: 1035 .

7. Gill IS: Retroperitoneal laparoscopic nephrectomy. Urol Clin North Am. 1998; 25: 343-60.

8. Dunn MD, Portis AJ, Shalhav AL, Elbahnasy AM, Heidorn C, McDougall EM, et al.: Laparoscopic versus open radical nephrectomy: a 9-year experience. J Urol. 2000; 164: 1153-9.

9. Abbou CC, Cicco A, Gasman D, Hoznek A, Antiphon P, Chopin DK, et al.: Retroperitoneal laparoscopic versus open radical nephrectomy. J Urol. 1999; 161: 1776-80.

10. Nelson CP, Wolf JS Jr: Comparison of hand assisted versus standard laparoscopic radical nephrectomy for suspected renal cell carcinoma. J Urol. 2002; 167: 1989-94.

11. Busby E, Das S, Rao Tunuguntla HS, Evans CP: Hand-assisted laparoscopic vs the open (flank incision) approach to radical nephrectomy. BJU Int. 2003; 91: 341-4.

12. Wolf JS Jr: Hand-assisted laparoscopy: pro. Urology. 2001; 58: 310-2.

13. Pareek G, Hedican SP, Gee JR, Bruskewitz RC, Nakada SY: Meta-analysis of the complications of laparoscopic renal surgery: comparison of procedures and techniques. J Urol. 2006; 175: 1208-13.

14. Matin SF, Dhanani N, Acosta M, Wood CG: Conventional and hand-assisted laparoscopic radical nephrectomy: Comparative analysis of 271 cases. J Endourol. 2006; 20: 891-4.

15. Venkatesh R, Belani JS, Chen C, Sundaram CP, Bhayani SB, Figenshau RS, et al.: Prospective randomized comparison of laparoscopic and hand-assisted laparoscopic radical nephrectomy. Urology. 2007; 70: 873-7.

16. Brown JA, Shah S, Siddiqi K, Boyd B: Incorporation of hand-assisted laparoscopic nephrectomy into an academic training program: an assessment of the util- 
ity of a 3-month minifellowship. J Laparoendosc Adv Surg Tech A. 2007; 17: 435-9.

17. Bandi G, Christian MW, Hedican SP, Moon TD, Nakada SY: Oncological outcomes of hand-assisted laparoscopic radical nephrectomy for clinically localized renal cell carcinoma: a single-institution study with $>$ or $=3$ years of follow-up. BJU Int. 2008; 101: 459-62.

18. Chung SD, Huang KH, Lai MK, Huang CY, Pu YS, Yu HJ, Chueh SC: Long-term follow-up of hand-assisted laparoscopic radical nephrectomy for organ-confined renal cell carcinoma. Urology. 2007; 69: 652-5.
Correspondence address:

Dr. Marcos Tobias-Machado

Rua Grauna, 104 / 131

São Paulo, SP, 04514-000, Brazil

Fax: + $55114437-3118$

E-mail: tobias-machado@uol.com.br

\section{EDITORIAL COMMENT}

This is an interesting prospective study comparing two laparoscopic approaches for radical nephrectomy: a retroperitoneoscopic (RRN) and hand-assisted (HALRN) approach, which attempts to define the best minimal access to this surgery. A shorter operative time and learning curve were in favor of HALRN versus a minor blood loss and smaller incision for organ retrieval in favor of RRN. No differences in the complication rate were observed. After a mean follow-up of 50 months, no difference in oncological control was found.

Although the study was conducted prospectively, a randomized trial would have been a better way, even if more difficult to perform, to compare different surgical techniques.

An important issue that should be investigated in any comparative surgical technique study is the health-related quality of life (HRQoL). In the literature, there is little reported data regarding longterm HRQoL after RRN and HALRN performed for renal cancer. Patel et al. (1) using the SF-36 questionnaire, showed no difference in terms of HRQoL between the two techniques with a mean follow-up of 6 months, but studies with a larger patient population and a longer follow-up are required.

We agree with the authors' statement that in general, both techniques are feasible for treatment of renal malignant tumors and the knowledge of both techniques should be theoretically familiar to the experienced surgeon.

However, our impression is that both techniques, as stated, are not equivalent. A difference of one hour in surgical time is the most striking difference described in this study and it should be considered an extremely important factor today in view of budget cuts for hospital costs, also considering that additional costs of laparoscopic hand-assisted devices could be avoided (2). In addition, longer anesthesia is associated with an increase in the incidence of perioperative complications and mortality (3).

Moreover, a shorter learning curve is another important factor to consider when we take in account the acceptance of a new technique and its use in the urological community outside teaching and referral hospitals. 
In search of the "Holy Grail" (i.e. the ideal minimally invasive method), this paper adds more information to the body of literature on laparoscopic radical nephrectomy. However, still today, the most important factor that guides the choice of a laparoscopic technique depends on the surgeon's experience and personal preference.

\section{REFERENCES}

1. Patel A, Wilson L, Blick C, Gurajala R, Rané A: Health-related quality of life after retroperitoneo- scopic and hand-assisted laparoscopic nephrectomy. J Endourol. 2005; 19: 849-52.

2. Ditonno P, Lucarelli G, Bettocchi C, Palazzo S, Palella GV, Battaglia M, et al.: "Deviceless" hand-assisted laparoscopic donor nephrectomy. Transplant Proc. 2008; 40: 1829-30.

3. Routh JC, Bacon DR, Leibovich BC, Zincke H, Blute ML, Frank I: How long is too long? The effect of the duration of anaesthesia on the incidence of non-urological complications after surgery. BJU Int. 2008; 102: 301-4.

Dr. Pasquale Ditonno \& Dr. Lucarelli Giuseppe Department of Emergency and Organ Transplantation Urology, Andrology and Kidney Transplantation Unit University of Bari Bari, Italy E-mail:ditonno@urologia.uniba.it 